quences of vancomycin-resistant enterococci in liver transplant patients. Transplantation 2001;72:1032-1037.

\section{VRE Colonization in Hemodialysis Patients}

Vancomycin-resistant enterococci (VRE) are increasing in prevalence at many institutions and are reported often in dialysis patients. Tokars and coinvestigators from the CDC's Hospital Infections Program studied the prevalence of, and risk factors for, VRE at seven outpatient hemodialysis centers (three in Baltimore, Maryland, and four in Richmond, Virginia). Rectal or stool cultures were performed on consenting hemodialysis patients during December 1997 to April 1998. Repeat cultures were obtained from consenting patients during May to July 1998 (median, 120 days later). Clinical and laboratory data and functional status ( 1 to 10 scale: 1 , normal function; 9 , home attendant, not totally disabled; 10 , disabled, living at home) were recorded.

Of 478 cultures performed, 20 (4.2\%) were positive for VRE. Among the seven centers, the prevalence of VREpositive cultures varied from $1.0 \%$ to $7.9 \%$. Independently significant risk factors for a VRE-positive culture were a functional score of 9 to 10 (odds ratio, $6.9 ; P<.001$ ), antimicrobial receipt within 90 days before culture (odds ratio, $6.1 ; P<.001)$, and a history of injection drug use odds ratio, 5.4; $P=.004)$.

The authors concluded that VRE-colonized patients were present at all seven participating centers, suggesting that careful infection control precautions should be used at all centers to limit transmission. In agreement with previous studies, VRE colonization was found more frequently in patients who had received antimicrobial agents recently, underscoring the importance of judicious antimicrobial use in limiting selection for this potential pathogen.

FROM: Tokars JI, Gehr T, Jarvis WR, Anderson J, Armistead N, Miller ER, et al. Vancomycin-resistant enterococci colonization in patients at seven hemodialysis centers. Kidney Int 2001;60:1511-1516.

\section{Community-Onset $S$ aureus Bacteremia}

Morin and Hadler, from the Epidemiology Program, Connecticut Department of Public Health, conducted a study that retrospectively analyzed the magnitude and epidemiology of community-onset Staphylococcus aureus (COSA) infections and methicillin-resistant $S$ aureus (MRSA) infections in four Connecticut metropolitan areas (population, 1.1 million). The study looked at hospital medical records of persons admitted with $S$ aureus bacteremia in 1998. COSA was categorized as "healthcare associated," "with underlying medical condition," or "no underlying medical condition."

Overall, $48 \%$ of $S$ aureus bacteremic infections were COSA (incidence, 17 cases $/ 100,000$ persons). Incidence increased with age and higher population density. In all,
$62 \%$ of infections were healthcare associated; $85 \%$ of the remaining cases had underlying medical conditions. MRSA accounted for $16 \%$ of healthcare-associated cases and cases with underlying conditions, but no cases with no underlying conditions. COSA bacteremic infections are as common as those due to pneumococci. MRSA is a well-established cause of COSA among persons at high medical risk for $S$ aureus infection.

FROM: Morin CA, Hadler JL. Population-based incidence and characteristics of community-onset Staphylococcus aureus infections with bacteremia in 4 metropolitan Connecticut areas, 1998. I Infect Dis 2001; 184:1029-1034.

\section{Low-Temperature Hydrogen Peroxide Plasma Sterilization of Bronchoscopes}

Bar and colleagues reported on a study to explore methods to prevent transmission of tuberculosis by bronchoscopes. Bronchoscopes were contaminated with Mycobacterium tuberculosis and decontaminated with a washer-disinfector (normal washing). Some were additionally disinfected with glutaraldehyde (intensive washing). Afterward, the bronchoscopes were sterilized using lowtemperature hydrogen peroxide plasma sterilization.

After normal washing, 8 of 17 samples had positive results by culture, and 7 of 17 had positive results by nucleic acid amplification technique. After intensive washing, all samples had negative results by culture, and 10 of 25 had positive results by the nucleic acid amplification technique. After sterilization with low-temperature hydrogen peroxide plasma sterilization, all samples had negative results by culture and the nucleic acid amplification technique.

The authors concluded that washing of bronchoscopes, as performed normally, is not sufficient for decontamination of bronchoscopes. Additional disinfection is recommended. If the nucleic acid amplification technique is used for diagnostic procedures, sterilization by low-temperature hydrogen peroxide plasma sterilization is recommended to avoid false-positive results.

FROM: Bar W, Marquez De Bar G, Naumann A, Rusch-Gerdes S. Contamination of bronchoscopes with Mycobacterium tuberculosis and successful sterilization by low-temperature hydrogen peroxide plasma sterilization. Am J Infect Control 2001;29:306-311.

\section{FDA Issues Draft Guidance on Blood- Donor Deferrals}

In an effort to reduce the risk of the human variant of mad cow disease, known as Creutzfeldt Jakob disease, the FDA recently issued a 35-page final guidance entitled "Revised Preventive Measures to Reduce the Possible Risk of Transmission of Creutzfeldt-Jakob Disease (CJD) and Variant Creutzfeldt-Jakob Disease (vCJD) by Blood and Blood Products." It recommends deferral of donors who 